\title{
The P-Median Problem in a Changing Network: The Case of Barcelona
}

\author{
Daniel Serra ${ }^{\dagger}$ \\ and \\ Vladimir Marianov
}

July 23, 1996

\begin{abstract}
In this paper a p-median-like model is formulated to address the issue of locating new facilities when there is uncertainty. Several possible future scenarios with respect to demand and/or the travel times/distance parameters are presented. The planner will want a strategy of positioning that will do as "well as possible" over the future scenarios. This paper presents a discrete location model formulation to address this P-Median problem under uncertainty. The model is applied to the location of fire stations in Barcelona
\end{abstract}

Key words: discrete facility location, scenarios, emergency services location

* Paper presented at the VII International Symposium on Locational Decisions, Edmonton, Canada. This project was partially funded by DCYGIT grant PB95-0980, Ministry of Education (Spain), and the Fire Protection Department of Barcelona

${ }^{\dagger}$ Dept. of Economics, Universitat Pompeu Fabra, Balmes 132, Barcelona, Spain. E-mail: serra@upf.es

‡The Catholic University of Chile 


\section{Introduction}

The P-median model, formulated by Hakimi in the mid-sixties (Hakimi 1964, 1965), has set the foundations of a myriad of location problems in the public and private sector. The basic problem is to find $\mathrm{P}$ locations which minimize the minimum average distance (or travel time) in a network. Only the nodes of the network need to be considered as locational candidates, since there is always at least one optimal solution that consists of the location of the facilities on the network nodes (see Hakimi 1965). The p-median model considers that the demand for service in each node and the travel times between nodes are deterministic.

The present paper recognizes uncertainty in:

1. the demand or population at the nodes of the network. That is, the demand or population to serve is not a known quantity but can assume different values depending on community growth or the community's economic vitality, among other factors; and/or

2. the different values that travel times can have depending on the time of the day, or day of the week.

These types of uncertainty are specially commom in an urban environment. For example, when locating fire stations in a city, travel times may drastically change during the day, and the population distribution might be very different due to commuting patterns. The CBD is usually very populated during the day, while in the evenings its population diminishes to the benefit of the residential areas. This situation implies that an optimal location of stations during the day can be very far from acceptable during the evenings. Therefore both uncertain situations have to be considered.

Several papers have addressed the issue of uncertainty in travel times or in demand. In general these models consider that travel time or demand follows 
a given distribution and some parameters are known, such as the mean and the deviation (see, among others, Weaver and Church, 1983, 1983b, 1984; Mirchandani and Odoni, 1979; Daskin and Hagani, 1984; Daskin, 1982, 1983 , 1987).

In this paper, uncertainty is treated by the classic scenario approach in which different patterns of demand and / or travel times are realized in different scenarios. The approach is two-pronged. First, over a range of possible demand scenarios, facilities are deployed to sites in such a way as to minimize

the maximum average travel time in a given scenario (MINMAX approach). Second, over that same range of scenarios, facilities are positioned in such a way to minimize the maximum regret. Regret is the difference between (1) the optimal average travel time that might have been obtained had the decision maker planned its sites for the scenario that actually occurred; and (2) the value of average travel time that was actually obtained (REGRET approach). A similar approach was used to locate facilities in a competitive environment

The rest of the paper is structured as follows: In the following section two p-median formulations are presented, corresponding to the MINMAX and REGRET approaches respectively. In the third section a heuristic method based on the Teitz and Bart (1968) procedure is developed. In the fourth section some computational experience is obtained and compared to the solutions using linear programming and branch and bound when necessary. Finally, a real application to the location of fire stations in Barcelona is presented.

\section{Problem Formulation}

Consider a spatial region that is represented by discrete points in a connected network. Each node can represent a town or a population area (e.g. census tracts) and has a parameter that can represent population, frequency of calls, or any other parameter relevant for the service to be located in that region. 
The problem now is that the extent of the efficiency depends not only on the location of the facilities but on the scenarios of demands and/or travel times that evolve over time.

As mentioned, two different objectives are proposed to obtain the final locations. The first one consists of the minimization of the maximum average distance that can be achieved across scenarios (MINMAX). The second one uses the regret approach, that is, minimize the maximum regret across scenarios (Regret).

The mathematical formulation of the model using the MINMAX Objective, based on the P-median Problem, is as follows:

The MinMaX P-Median Problem

$$
\begin{array}{rlr}
\min M & & \\
\text { s.t. } & & \\
\sum_{i \in I} \sum_{j \in J} \frac{a_{i k} d_{i j}^{k}}{W_{k}} x_{i j}^{k} \leq M & & \\
\sum_{j \in J} x_{i j}^{k} & =1 & \\
w_{j} \geq x_{i j}^{k} & i \in I, \quad j \in J, \quad k \in K
\end{array}
$$

where 
$i, I \quad$ Index and set of demand nodes

$j, J \quad$ Index and set of potential facility sites

$k, K$ Index and set of scenarios

$a_{i k} \quad$ Population at node $i$ in scenario $k$

$W_{k} \quad$ Total population in scenario $k$

$d_{i j}^{k} \quad$ Travel time between $i$ and $j$ in scenario $k$

$p \quad$ Number of services to locate

$x_{i j}^{k} \quad 1$, if node $i$ is assigned to node $j$ in scenario $k$

0 , otherwise

$w_{j} \quad 1$, if there is a facility at $j ; 0$, otherwise

The first set of constraints is directly related to the objective. Since we want to minimize the maximum average travel time across scenarios, we want to find a set of locations that will give the smallest maximum average travel time possible when evaluated for all scenarios. The left side of each constraint (one for each scenario) represents the total travel time that will be achieved in the corresponding scenario. The right hand side, $M$, is the same in each constraint. Of course, the $M$ is shifted to the left hand side of each constraint in the process of solution since $M$ is an unknown. The objective of the model is to minimize $M$. That is, the model will try to find a set of locations that minimizes the highest total travel time achieved when accounted for all scenarios.

The rest of the constraints are very similar to the constraint set of the p-median problem. The main difference is that now $x_{i j}^{k}$ assignment variables are used and we also need to add locational variables $w_{j}$. The second set of constraints (3) defines that for each scenario, demand area $i$ has to assign to only one potential facility node $j$. The third set of constraints (4) will determine, again for each scenario, if assignement can be achieved; that is, demand node $i$ may assign to node $j$ in scenario $k$ if, and only if, there is a facility at $j$. Finally, the last constraint (5) sets the number of facilities needed to be located.

If the regret objective is used, constraint set (2) is replaced by the following 
set:

$$
\sum_{i \in I} \sum_{j \in J} \frac{a_{i k} d_{i j}^{k}}{W_{k}} x_{i j}^{k}-Z_{k} \leq M \quad k \in K
$$

where $Z_{k}$ is the optimal objective, a known value, found when Firm A locates $p$ facilities in each scenario independently. It is found by applying the original p-median formulation to each scenario individually. The unkown variable $M$ in this case represents the largest regret evaluated over all scenarios. Therefore, the new objective remains to minimize $M$, that is, we seek to minimize the maximum regret overall scenarios. For a given set of locations, the left side of each one of the constraints (there is again one constraint for each scenario) computes the difference between the optimal total travel time that can be achieved in the scenario, if the locations were optimized for just that scenario; and the total travel time given the chosen set of locations, that is, the regret. The objective improves as the largest regret found in a given scenario is reduced. The final solution will be found when the minimum regret is obtained over all scenarios in a given scenario for a given set of locations. The rest of the constraints remain the same.

\section{Solution Method}

As in most p-median based models, in general it is necessary to develop a heuristic method to tackle the problem, due to the large number of variables and constraints that arise for a medium size network. This is especially true for the formulations presented in this paper due to the extremely large number of variables and constraints involved and to the nature of constraints (2) in the MINMAX p-median problem and constraints (2') in the Regret problem. These constraints are non integer friendly, and the use of relaxed linear programming and branch and bound when necessary might be computationally burdensome. 
In this paper an exchange heuristic is developed to solve both formulations. The heuristic algorithm is based on the well known one-opt Teitz and Bart procedure and involves two phases. In the first one, an initial solution is obtained using the P-median problem. In the second phase a one-opt trade is used to try to improve the initial objective.

A good starting solution (phase 1) can be obtained as follows: for each scenario a P-median problem is used to find the optimal location of Firm A's servers. Once the the optimal locations are obtained for each individual scenario, they can be used to compute the average travel time that could be achieved in the other scenarios. This can be represented in a matrix form, where each row represents a given scenario, and each column represents the average travel time that is achieved given the optimal locations given in each scenario (row). For example, the value of the matrix element $c_{i j}$ (that is row $i$, column $j$ ) corresponds to the average travel time that would be achieved in scenario $j$ if the optimal locations in scenario $i$ were implemented. Therefore, the coefficients in the diagonal represent the minimum average travel time that can be achieved in each scenario. Once the table is obtained, the regret from optimal capture can be computed for each scenario. Therefore, initial locations for the heuristic can be choosen depending on the objective used. If the Minmax objective is used, the initial locations will correspond to scenario (row) $i$ where the largest $c_{i j}$ is minimum. If the regret objective is used, the initial solution will correspond to the locations in scenario $i$ where the largest regret is at minimum. Therefore, for each scenario $j$ the regret from optimal capture is computed, and we choose the scenario $i$ that gives the largest regret. The locations corresponding to the scenario $i$ where the largest regret is minimum will define the initial solution.

Once the initial locations are obtained, the second phase of the heuristic algorithm is used to improve the objective. At each iteration one server's location is traded. The new objective is computed and that will be stored as the best solution so far, if it leads to an improvement. Otherwise, the 
relocation will be ignored and the previous solution is restored. The one-opt trade will be done for all nodes and facilities. A step-by-step description of the algorithm when the Minmax objective is used follows:

1. For each scenario, find the locations of $p$ facilities where the minimum average time is achieved (a P-median problem is solved for each scenario).

2. Compute, again for each scenario, the average travel time that is achieved for all the location patterns (one pattern for each scenario) found in step 1.

3. Choose, as a starting set of locations, those whose maximum average travel time across all scenarios is the smallest.

4. Trade the location of one of the $p$ facilities.

5. Compute the new average travel times achieved in each scenario. When the Minmax objective is used, if the maximum average travel time across all scenarios is smaller than before the trade, keep the solution. If not, restore the old solution. Repeat steps 4-5 until all facilities and nodes have been exchanged in a complete cycle of trading.

6. If the objective after steps 4-5 has improved, go to step 4 and restart the procedure. When no improvement is achieved on a complete set of one-at-a-time trades, stop.

For the regret objective, the heuristic is modified as follows:

1. For each scenario, find the locations of $p$ facilities where the minimum average travel time is achieved (a P-median problem for each scenario).

2. Compute, again for each scenario, the average travel time that is achieved for all the location patterns (one pattern for each scenario) found in step 1. 
3. Find, for each scenario $j$, the regret from optimally locating $p$ facilities in all scenarios. For each scenario $i$ choose the scenario $j$ that would give the maximum regret. The initial solution -the initial locations and regret- will be the scenario where the largest regret is minimum.

4. Trade the location of one of the $p$ facilities.

5. Compute the new average travel times and the regrets that are achieved in each scenario. Choose the locations corresponding to the scenario that gives the smallest maximum regret over the other scenarios. If this regret is smaller than before the one-opt trade, store the solution. If not, restore the old locations and objective. Repeat steps 4-5 until all of Firm A's facilities and nodes have been traded.

6. If the objective after steps $4-5$ has improved, go to step 4 and restart the procedure. When no improvement is achieved on a complete set of one-at-a-time trades, stop.

\section{Computational Experience}

First, 10 different 20-node networks were used with four different travel time scenarios each. Node coordinates for each network were generated randomly in a $100 \times 100$ grid. The euclidean distance was used to compute travel time. These times correspond to the first scenario. Then, for the rest of the scenarios, these travel times were modified randomly, allowing a maximum of a $50 \%$ deviation from original travel times. The population in each node was computed following the same process. For the first scenario it was computed randomly. Then, for the rest of the scenarios, the population of each node were random deviations from the ones in the first scenario, allowing a maximum deviation of $50 \%$.

LINGO, a linear, integer and nonlinear programming software package was used on a HP Apollo 710 workstation with a Risc Processor. Both the 
Table 1: Results, $\mathrm{LP}+\mathrm{BB}$ and Heuristics

\begin{tabular}{|c|c|c|c|c|c|c|c|c|}
\hline \multirow[b]{2}{*}{$\begin{array}{l}\text { Sce- } \\
\text { nario }\end{array}$} & \multicolumn{4}{|c|}{ MINMAX } & \multicolumn{3}{|c|}{ REGRET } & \\
\hline & Time & $\begin{array}{c}\# \text { of } \\
\text { Brnchs }\end{array}$ & $\begin{array}{r}\mathrm{Obj} . \\
\mathrm{LP}+\mathrm{BB}\end{array}$ & $\begin{array}{l}\text { value } \\
\text { HEUR }\end{array}$ & Time & $\begin{array}{c}\# \text { of } \\
\text { Brnchs }\end{array}$ & $\begin{array}{r}\mathrm{Obj} \cdot \\
\mathrm{LP}+\mathrm{BB}\end{array}$ & $\begin{array}{l}\text { value } \\
\text { HEUR }\end{array}$ \\
\hline 1 & $19: 28$ & 27 & 18.81 & 19.25 & 27.41 & 65 & 2.98 & 2.98 \\
\hline 2 & $16: 45$ & 7 & 18.14 & 18.30 & 13.22 & 10 & 3.37 & 3.37 \\
\hline 3 & $16: 02$ & 63 & 17.88 & 17.88 & 17.34 & 48 & 2.54 & 3.18 \\
\hline 4 & $26: 17$ & 105 & 18.19 & 18.19 & 23.31 & 80 & 2.23 & 2.49 \\
\hline 5 & $16: 37$ & 58 & 17.31 & 17.31 & 26.29 & 94 & 1.78 & 1.78 \\
\hline 6 & $15: 49$ & 90 & 18.75 & 18.75 & 19.47 & 84 & 3.20 & 3.54 \\
\hline 7 & $12: 04$ & 41 & 18.92 & 18.92 & 17.40 & 27 & 2.54 & 2.60 \\
\hline 8 & $8: 05$ & 28 & 17.96 & 17.96 & 21.41 & 52 & 2.44 & 3.61 \\
\hline 9 & $16: 17$ & 76 & 18.64 & 18.64 & 30.03 & 97 & 2.62 & 3.72 \\
\hline 10 & $19: 28$ & 70 & 18.42 & 18.64 & 24.12 & 67 & 2.51 & 2.51 \\
\hline
\end{tabular}

p-median problems for each scenario and the MINMAX and Regret p-median problems were solved on this network using standard Linear Programming with Branch and Bound when necessary $(\mathrm{LP}+\mathrm{BB})$. The heuristics were programmed in FORTRAN 77, using the initial optimal locations for each scenario found with LP+BB. Results are presented in Table 1.

The first column refers to the problem number. The second and sixth columns refer to the $\mathrm{CPU}$ time (in minutes) used by the computer when using LP-BB in each model specification respectively. The third and seventh columns indicate the number of branches needed in the branch and bound procedure to obtain optimal integer solutions for both models. The objective value of the MINIMAX model when using $\mathrm{LP}+\mathrm{BB}$ and the Heuristic are in columns four and five respectively. Finally, in columns eight and nine the solutions for the Regret model are presented.

Both models required a considerable amount of time to solve when using the LP-BB procedure. The average time was 16.53 minutes for the MINIMAX model and 22.00 minutes for the REGRET model. These high values are due 
to the large number of branches needed to obtain integer optimal solutions. None of the problems solved gave integer values using the linear programming relaxation approach. On the other hand, the time used by both heuristics was almost insignificant. All problems were solved in less than 2 seconds of CPU time. The computational performance of the heuristic was quite satisfying for the MINMAX model since 7 out of 10 problems obtained the optimal solution. The Regret approach performed less well, since only 4 optimal solutions were obtained. On the other hand, in problems 8 and 9 the performance was quite deceiving, since the objective value was far from optimal.

\section{An Application to the Location of Fire Sta- tions in Barcelona}

The city of Barcelona is planning to relocate its fire stations. Actually, there are 7 fire stations, with only 4 of them fully equiped. Their actual location is less than desirable, since their siting criteria correspond to historical reasons and the newest on dates from the 1960's. Due to the morphological and traffic volumes changes in the city, actual travel times could be greatly improved by relocating stations. The city is planning to relocate some of them, and expects to maintain the actual service quality with only four stations. In this section a preliminary study of the optimal siting is addressed using the concepts of MINMAX and Regret.

Barcelona is divided into 201 transportation zones (see Figure 1). For each zone, both population and estimated average travel times between them are known for a given interval of the day. Seven different intervals were considered in a 24-hour day, i.e., there are seven scenarios to be considered. The time intervals are shown in Table 2 .

First, for each scenario Rosing and ReVelle's one-opt heuristic was applied to obtain the optimal locations. This solution method is based on the Teitz 
Table 2: Time intervals for Barcelonas's 201-node network

\begin{tabular}{|c|c|c|c|c|c|c|}
\hline SCEN1 & SCEN2 & SCEN3 & SCEN4 & SCEN5 & SCEN6 & SCEN7 \\
\hline 12AM-5AM & 5AM-9AM & 9AM-12PM & 12PM-3PM & 3PM-6PM & 6PM-10PM & 10PM-12AM \\
\hline
\end{tabular}

Table 3: Initial results accross scenarios: MINMAX and Regret

\begin{tabular}{|l|r|rrrrrrr|}
\hline & $\begin{array}{c}\text { Optimal } \\
\text { Locations }\end{array}$ & 1 & 2 & 3 & 4 & 5 & 6 & 7 \\
\hline SCEN1 & 58,126, & $\mathbf{3 . 0 8}$ & 3.11 & 4.45 & 3.27 & 3.21 & 3.46 & 2.96 \\
& 161,178 & $(0.00)$ & $(0.08)$ & $(0.244)$ & $(0.06)$ & $(0.04)$ & $(0.15)$ & $(0.07)$ \\
\hline SCEN2 & 58,124, & 3.18 & $\mathbf{3 . 0 3}$ & 4.52 & 3.38 & 3.27 & 3.42 & 3.03 \\
& 159,173 & $(0.10)$ & $(0.00)$ & $(0.31)$ & $(0.16)$ & $(0.11)$ & $(0.11)$ & $(0.13)$ \\
\hline SCEN3 & 84,126 & 3.25 & 3.21 & $\mathbf{4 . 2 1}$ & 3.38 & 3.30 & 3.45 & 3.15 \\
& 154,169 & $(0.16)$ & $(0.17)$ & $(0.00)$ & $(0.17)$ & $(0.13)$ & $(0.15)$ & $(0.26)$ \\
\hline SCEN4 & 58,126, & 3.17 & 3.18 & 4.67 & $\mathbf{3 . 2 1}$ & 3.28 & 3.56 & 3.02 \\
& 162,179 & $(0.09)$ & $(0.14)$ & $(0.46)$ & $(0.00)$ & $(0.11)$ & $(0.26)$ & $(0.12)$ \\
\hline SCEN5 & 58,126, & 3.15 & 3.13 & 4.47 & 3.30 & $\mathbf{3 . 1 7}$ & 3.44 & 2.99 \\
& 155,169 & $(0.07)$ & $(0.09)$ & $(0.26)$ & $(0.08)$ & $(0.00)$ & $(0.13)$ & $(0.10)$ \\
\hline SCEN6 & 10,91, & 3.23 & 3.15 & 4.59 & 3.36 & 3.23 & $\mathbf{3 . 3 0}$ & 3.16 \\
& 145,155 & $(0.15)$ & $(0.12)$ & $(0.38)$ & $(0.15)$ & $(0.07)$ & $(0.00)$ & $(0.27)$ \\
\hline SCEN7 & 58,124, & 3.14 & 3.11 & 4.59 & 3.26 & 3.28 & 3.46 & $\mathbf{2 . 8 9}$ \\
& 147,161 & $(0.06)$ & $(0.08)$ & $(0.378)$ & $(0.05)$ & $(0.11)$ & $(0.16)$ & $(0.00)$ \\
\hline
\end{tabular}

and Bart heuristic and has two phases. In the first phase, 200 initial solutions (obtained from 200 diferent initial locations) were generated to obtain the set of potential facility nodes to be used in the second phase of the heuristic. In this second phase, $\mathrm{LP}+\mathrm{BB}$ was used to obtain the final solution of the p-median problem.

Once the optimal locations are known for each scenario, these were used to obtain the average time that would be achieved in the other scenarios. Results are shown in Table 3. Optimal objective values are shown in bold face. Values in parenthesis represent the regret in each scenario.

Table 3 can be used to compute the initial locations and objective (phase 1 of the algorithms). Rows SCEN1, SCEN2, SCEN3, SCEN4, SCEN5, SCEN6 and SCEN7 in the table correspond to the average travel time obtained when the servers are located optimally with the p-median problem in scenarios 1 to 
Table 4: Final results: MINMAX and Regret

\begin{tabular}{|l|r|rrrrrrr|}
\hline & $\begin{array}{r}\text { Optimal } \\
\text { Locations }\end{array}$ & 1 & 2 & 3 & 4 & 5 & 6 & 7 \\
\hline MINMAX & 58,126, & 3.17 & 3.18 & $\mathbf{4 . 6 7}$ & 3.21 & 3.28 & 3.56 & 3.02 \\
& 162,179 & $(0.09)$ & $(0.14)$ & $(0.46)$ & $(0.00)$ & $(0.11)$ & $(0.26)$ & $(0.12)$ \\
\hline REGRET & 49,82 & 3.23 & 3.13 & 4.42 & 3.41 & 3.27 & 3.50 & 3.10 \\
& 126,159 & $(0.15)$ & $(0.10)$ & $(0.21)$ & $(\mathbf{0 . 2 0})$ & $(0.11)$ & $(0.19)$ & $(0.21)$ \\
\hline
\end{tabular}

7 respectively. In parenthesis the regret from the optimal total travel time is computed.

From this table we can now choose our starting solution for both objectives. If the MINMAX objective is used, the initial solution will correspond to the optimal locations in scenario 2 (see Table 2 ) and the value of the objective $m$ will be equal to 1899 , since it currently maximizes the minimum capture across scenarios. If the regret scenario is used, the initial objective $U$ equal to 292.0 , that is, the smallest maximum regret that can be achieved across scenarios, given the optimal locations in scenarios 1 to 4 . Therefore the initial locations correspond to the ones found when locating optimally the second scenario. Once the initial conditions are established, the second phase is used to improve the objective. Standard FORTRAN 77 was used to implement the heuristic. Results are presented in Table 4.

The value of the objective $m$ found for the MINMAX model is equal to $4.67 \mathrm{mn}$ (average travel time), and the final locations are 58,126,162 and 179 . If the regret model is used, the final objective $U$ is equal to 0.20 and final locations are 49, 82, 126 and 159. For each row, the total travel time obtained for each scenario is computed from the locations obtained. For example. if the MINMAX approach is used, the average travel time will be 3.21 in scenario 4.

The second phase of the heuristic did find a better set of locations for the Regret objective. It did not, however, obtain a better optimal solution found using linear programming plus branch and bound. This was expected, 
since scenario 4 corresponds to peak traffic volumes and consequently average travel times are much higher than the ones in the other scenarios.

\section{Conclusions}

A formulation has been presented to tackle the issue of uncertainty in locating emergency services, where it is possible to define different scenarios that account for differences in travel times and/or the demand in the region of interest. Two different objectives were used: minimize the maximum average travel time across scenarios (MINMAX objective) and minimize the maximum regret across scenarios (regret objective). Both an optimal solution method $(\mathrm{LP}+\mathrm{BB})$ and a heuristic algorithm were described and used in an example. Then, the model was applied to the location of fire stations in the city of Barcelona.

Finally, observe that in the models presented each scenario is equally weighted. Both MINMAX and Regret objectives can be modified to account for the situation where scenarios have different weights (for example, if the time length the scenario is significantly diferent. In this case, constraints (2) and $\left(2^{\prime}\right)$ can be modified as follows:

$$
\begin{gathered}
\beta_{k}\left(\sum_{i \in I} \sum_{j \in J} \frac{a_{i k} d_{i j}^{k}}{W_{k}} x_{i j}^{k}-Z_{k}\right) \leq M \quad k \in K \\
\beta_{k} \sum_{i \in I} \sum_{j \in J} \frac{a_{i k} d_{i j}^{k}}{W_{k}} x_{i j}^{k} \leq M \quad k \in K \quad\left(6^{\prime}\right)
\end{gathered}
$$

where $\beta_{k}$ corresponds to the weight assigned to scenario $k$. The corresponding heuristic methods can be easily modified to account for the weights. 


\section{References}

Daskin, M. (1982). Application of an expected covering model to ems system design. Decision Siences, 13(3):416-439.

Daskin, M. (1983). A maximum expected covering location model: Formulation, properties, and heuristic solution. Transportation Science, 17:48-70.

Daskin, M. (1987). Location, dispatching and routing models for emergency services with stochastic travel times. In Gosh, A. and Rushton, G., editors, Spatial Analysis and Location-Allocation Models, pages 224-265. Van Nostrand Reinhold Co., New York.

Daskin, M. and Hagani, A. (1984). Multiple vehicle routing and dispatching to an emergency scene. Environment and Planning A, 16:1349-1359.

Hakimi, S. (1964). Optimum location of switching centers and the absolute centers and medians of a graph. Operations Research, 12:450-459.

Hakimi, S. (1965). Optimum location of switching centers in a communications network and some related graph theoretic problems. Operations Research, 13:462-475.

Mirchandani, P. and Odoni, A. (1979). Locations on medians on stochastic networks. Transportation Science, 13:85-97.

Serra, D. and ReVelle, C. (1996). The maximum capture problem with uncertainty. Environment and Planning B, 62:49-59.

Tietz, M. B. and Bart, P. (1968). Heuristic methods for estimating the generalized vertex median of a weighted graph. Operations Research, 16:955965.

Weaver, J. and Church, R. (1983a). Computational procedures for location problems on stochastic networks. Transportation Science, 17:168-180. 
Weaver, J. and Church, R. (1983b). A median facility location problem with non closest facility service. Working Paper 83-011, Dept. of Management Sciences and Statistics, College of Commerce and Business Administration, University of Alabama.

Weaver, J. and Church, R. (1984). The vast median facility location model. Working Paper 84-02, Dept. of Management Sciences and Statistics, College of Commerce and Business Administration, University of Alabama. 
In this paper a p-median-like model is formulated to address the issue of locating new facilities when there is uncertainty. Several possible future scenarios with respect to demand and/or the travel times/distance parameters are presented. The planner will want a strategy of positioning that will do as "well as possible" over the future scenarios. This paper presents a discrete location model formulation to address this P-Median problem under uncertainty. The model is applied to the location of fire stations in Barcelona 